\title{
Stereotyped Axon Pruning via Plexin Signaling Is Associated with Synaptic Complex Elimination in the Hippocampus
}

\author{
Xiao-Bo Liu, ${ }^{1 \star}$ Lawrence K. Low, ${ }^{1 \star}$ Edward G. Jones, ${ }^{1,2}$ and Hwai-Jong Cheng ${ }^{1,3}$ \\ ${ }^{1}$ Center for Neuroscience, ${ }^{2}$ Department of Psychiatry, and ${ }^{3}$ Section of Neurobiology, Physiology, and Behavior, ${ }^{3}$ Department of Pathology and Laboratory \\ Medicine, University of California, Davis, Davis, California 95616
}

\begin{abstract}
Plexin signaling is required for stereotyped pruning of long axon collaterals in the vertebrate CNS; however, a cellular basis for plexins on stereotyped pruning has not been determined. Using quantitative electron microscopy and immunocytochemistry, we found that infrapyramidal mossy fiber axon collaterals form transient synaptic complexes with basal dendrites of CA3 pyramidal cells in the early postnatal mouse hippocampus. At later postnatal ages, these synaptic complexes stop maturing and are removed before stereotyped pruning by a mechanism that does not involve axon degeneration and glial cell engulfment. In knock-out mice that lack plexin-A3 signaling, the synaptic complexes continue to mature, and, as a result, the collaterals are not pruned. Thus, our results suggest that intact plexin-A3 signaling contributes to synaptic complex elimination, which is associated with stereotyped axon pruning.
\end{abstract}

Key words: axon pruning; plexin; semaphorin; synaptic complex; mossy fiber; hippocampus; granule cell

\section{Introduction}

In the CNS, the construction of precise circuitry is often preceded by a stage of development that involves the formation of collateral branch projections off an axon to ensure proper coverage of most potential synaptic targets (Purves and Lichtman, 1980; O'Leary, 1987; O’Leary et al., 1990; Kantor and Kolodkin, 2003; Luo and O'Leary, 2005). As a consequence, some of the long collateral projections that extend to transient but inappropriate locales are selectively removed in a stereotyped manner to ensure that proper targeting of axons is maintained.

Many aspects of long-range stereotyped pruning have distinguished it from other forms of pruning that occur stochastically within the target region such as those observed at the neuromuscular junction (NMJ) and the cerebellum (Lohof et al., 1996; Bernstein and Lichtman, 1999; Sanes and Lichtman, 1999; Lichtman and Colman, 2000). In stereotyped pruning, the removal of subsets of inappropriately targeted long axon collaterals is often predictable because the transient branches can be identified before their removal (Chalupa and Killackey, 1989; O'Leary et al., 1990; Weimann et al., 1999; Lee et al., 2000; Bagri et al., 2003; Kantor and Kolodkin, 2003; Watts et al., 2003). In addition, the length of axon collaterals that undergo process elimination in stereotyped pruning often exceeds hundreds of micrometers (Chalupa and Killackey, 1989; O’Leary et al., 1990; Bagri et al.,

\footnotetext{
Received June 27, 2005; revised Aug. 16, 2005; accepted Aug. 22, 2005

This research was supported by grants from the Whitehall Foundation, the Klingenstein Fund, the Sloan Foundation, the Medical Investigation of Neurodevelopmental Disorders Institute, and by National Institutes of Health Grant HD045757 (H.-J.C.). We thank J. Wenzel, P. Schwartzkroin, K. McAllister, L. Chalupa, S. Hayes, M. Lucanic, and members of the Cheng laboratory and the Jones laboratory for valuable comments and technical support.

${ }^{*}$ X.-B.L. and L.K.L. contributed equally to this work.

Correspondence should be addressed to Hwai-Jong Cheng, Center for Neuroscience, University of California, Davis, 1544 Newton Court, Davis, CA 95616. E-mail: hjcheng@ucdavis.edu.

DOI:10.1523/JNEUROSCI.2648-05.2005

Copyright $\odot 2005$ Society for Neuroscience $\quad$ 0270-6474/05/259124-11\$15.00/0
}

2003); in contrast, the pruning of terminal arbors at the NMJ and the cerebellum (Lohof et al., 1996; Sanes and Lichtman, 1999; Lichtman and Colman, 2000) involves a few micrometers of branches.

Although much effort has concentrated on molecular pathways that regulate stereotyped pruning in the vertebrate CNS, less is known about the cellular mechanisms that contribute to the predictable loss of inappropriately targeted long axon collaterals. Until recently, two cellular mechanisms have been thought to account for the disappearance of exuberant axon collaterals during pruning. Presumably, axon collaterals could just retract in a distal to proximal manner, whereas the axonal contents are recycled to other parts of the axon (Riley, 1981); however, no direct evidence has been shown to account for this in the vertebrate CNS. Alternatively, the axon collaterals may degenerate in a process resembling classic Wallerian degeneration (Jones and Powell, 1969; Rosenthal and Taraskevich, 1977) as was observed for stereotyped pruning in Drosophila (Watts et al., 2003, 2004; Broadie, 2004; Marin et al., 2005). At the vertebrate NMJ, however, it seems that neither retraction nor classic Wallerian degeneration was observed (Bishop et al., 2004). Instead, in a third mechanism that was termed "axosome shedding," retreating axons were reportedly removed via shedding of contents from the axon terminal and the axonal remnants that were left behind were actively engulfed by Schwann cells. Because of the unique characteristics of long axon collaterals that are stereotypically removed in the vertebrate CNS, it is still unclear whether the same cellular mechanisms (retraction, axosome shedding, or classic Wallerian degeneration) could be involved in stereotyped pruning.

To address these issues, we focused our attention on an area in the CNS, the hippocampal mossy fiber system, in which longrange stereotyped pruning could be visualized and studied in detail. In the mouse hippocampus, plexin signaling regulates the 
stereotyped removal of mossy fiber collaterals (Bagri et al., 2003). Recently, we showed that plexin-A3 (PLXA3) signaling with neuropilin-2 (NPN-2) for semaphorin-3F (Sema3F) was required for the stereotyped pruning of mossy fiber axon collaterals (Bagri et al., 2003). A cellular basis for this pruning event, however, is not known. We, therefore, sought to determine a cellular mechanism for stereotyped pruning in the vertebrate CNS by focusing on cellular events that were occurring in vivo as the mossy fibers were being pruned.

We report here that the mossy fibers of the infrapyramidal bundle (IPB) of the hippocampus can establish synaptic contacts with the dendrites of CA3 pyramidal cells in a structure called a "synaptic complex" (see below). Their fibers are pruned during peak synaptic elimination. Because no ultrastructural evidence of axon degeneration or glial cell involvement was observed, we conclude that the axons are pruned by axon retraction. Finally, intact plexin signaling is required for synaptic complex elimination that occurred before axon pruning.

\section{Materials and Methods}

Mouse breeding. Animal protocols were approved by the Institutional Animal Care and Use Committee at University of California, Davis. Genotyping was performed as described previously (Chen et al., 2000; Cheng et al., 2001).

Immunoelectron microscopy. Wild-type (WT), PLXA3 ${ }^{-/-}$, and NPN$2^{-/-}$knock-out $(\mathrm{KO}) \mathrm{CD}-1$ mice at different postnatal ages [postnatal day 10 (P10), P15, P20, P25, P35, P45, and P65] were used for immunoelectron microscopic (immuno-EM) study. A minimum of two animals were studied for each phenotype and age group. Animals were anesthetized with an overdose of Nembutal and perfused transcardially with cold saline followed by $4 \%$ paraformaldehyde plus $0.5-3 \%$ glutaraldehyde in $0.1 \mathrm{M}$ phosphate buffer. Brains were postfixed several hours in $4 \%$ paraformaldehyde in $0.1 \mathrm{M}$ phosphate buffer at $4^{\circ} \mathrm{C}$ and sectioned at $50-70$ $\mu \mathrm{m}$ with a Leica (Blackburn, IL) VT1000 S vibrating microtome. Sections were collected and stored in ice-cold $0.1 \mathrm{M}$ phosphate buffer.

The immunocytochemical staining procedure has been described previously in detail (Liu and Jones, 2003). Each set of experiments including wild-type and $\mathrm{KO}$ samples at different postnatal ages was processed simultaneously for immunocytochemistry using specific antibodies. Briefly, sections were preincubated in normal blocking serum and then placed in the following primary antibodies for $48 \mathrm{~h}$ at $4^{\circ} \mathrm{C}$ : anticalbindin-28K (CB) (diluted 1:2000, a polyclonal antibody raised in rabbit; Chemicon, Temecula, CA), anti-NMDA receptor subunit 1 (NMDAR1) (1:300, a rabbit polyclonal antibody; Chemicon), antivesicular glutamate transporter 1 (VGLUT1) (1:5000, a guinea pig polyclonal antibody; Chemicon), and anti-VGLUT2 (1:5000, a guinea pig polyclonal antibody; Chemicon). After washing in phosphate buffer, sections were incubated in a solution containing biotinylated secondary antibodies (1:200; Vector Laboratories, Burlingame, CA) and further processed using an $\mathrm{ABC}$ kit. Immunoreactivity was visualized by a $\mathrm{DAB}$ reaction. Dense immunolabeled sections were selected for electron microscopy. Sections were osmicated in $1 \% \mathrm{OsO}_{4}$ and dehydrated in grade ethanol and acetone and then flat embedded in Araldite. Serial ultrathin sections $(70-80 \mathrm{~nm})$ were cut on a Leica Ultramicrotome and were collected on Formvar-coated single-slot grids (EMS, Hatfield, PA). Sections were lightly stained with uranyl acetate and lead citrate and examined in a Philips CM120 electron microscope at $80 \mathrm{kV}$. Electron microscopic images were acquired by a $2000 \times 2000$ high-resolution CCD camera attached to the microscope (Gatan, Pleasanton, CA). Images were processed using an EM software program (DigitalMicrograph; Ga$\tan$ ) and composed in Photoshop 6.5 (Adobe System, San Jose, CA). For three-dimensional (3D) reconstruction, serial EM images were traced, reconstructed in Reconstruct 2.4 (http://synapses.bu.edu, Boston University), and rendered using 3D Studio Max (Discreet, Montreal, Quebec, Canada).

Data analysis for immunoelectron microscopy. All electron micrographs for WT and KO mice were assigned a number and randomized before analysis. A minimum of 15 mossy fiber synaptic complexes were analyzed for each phenotype and age group, except in P35 WT mice $(n=10$ synaptic complexes analyzed) in which most synaptic complexes and fibers had been pruned back to the dentate gyrus. Quantitative assessments for mossy fiber bouton perimeter, postsynaptic density (PSD) length, number of spines per bouton, and number of asymmetric contacts per bouton were measured using Scion (Frederick, MD) Image 4.0.2. Statistics for all parameters were obtained from Statistica 6.0 (StatSoft, Tulsa, OK).

Overall assessment for the staging of mossy fiber synaptic complexes was based on a previous reported study on the postnatal development of main bundle (MB) mossy fiber boutons over time (Amaral and Dent, 1981). Mossy fiber synaptic complexes were classified as stage 1 if they contained mossy fiber boutons that resembled MB boutons at $\mathrm{P} 3$ with a small perimeter $(<4.0 \mu \mathrm{m})$ and contained one or no synaptic contacts. The boutons in these synaptic complexes were circular in shape and lacked invaginations from CA3 dendritic spines. Most synaptic contacts of boutons in stage 1 synaptic complexes were on dendritic shafts, whereas a few were observed on small dendritic spines. Mossy fiber synaptic complexes were classified as stage 2 if they contained mossy fiber boutons that resembled $\mathrm{MB}$ boutons at either $\mathrm{P} 9$ or $\mathrm{P} 14$ that tended to be larger in appearance (between 5 and $9 \mu \mathrm{m}$ in perimeter). The number of synaptic contacts with boutons in stage 2 synaptic complexes was more abundant (two to three synapses) compared with boutons in stage 1 synaptic complexes; a majority of the synapses were made on CA3 dendritic shafts adjacent to the peripheral edges of the bouton. The boutons in these synaptic complexes were also quite circular in appearance compared with boutons in stage 1 synaptic complexes. Mossy fiber synaptic complexes were classified as stage 3 if they contained mossy fiber boutons that resembled MB boutons at P21 and P35. The boutons in these synaptic complexes were not only larger ( $>9 \mu \mathrm{m}$ in perimeter) compared with boutons at stage 2 , but they were also more complex in shape: they tended to be less circular, a characteristic likely attributed to multiple invaginations of $\mathrm{CA} 3$ dendritic spines (two to four invaginations from CA3 spines per bouton) within the central regions of the boutons. Compared with stage 2 , the number of stage 3 mossy fiber bouton synapses on CA3 dendritic spines (more than three synapses on different spines) outnumbered the number of synapses on CA3 dendritic shafts (one to two synapses on different shafts).

Parallel Timm's and anti-calbindin-28K labeling. WT and PLXA3 knock-out mice at different postnatal ages (P15-P30) were anesthetized with an overdose of Nembutal and perfused transcardially with a buffered sulfide solution containing $0.4 \% \mathrm{Na}_{2} \mathrm{~S}$. This was followed by perfusion with ice-cold $4 \%$ paraformaldehyde. The brains were removed, postfixed overnight, and sectioned on a Leica VT1000 S vibrating microtome. Coronal sections, $40 \mu \mathrm{m}$, of the dorsal hippocampus were then collected in serial manner. Timm's method for staining for zinc ions within the mossy fiber boutons was applied to one set of alternate serial sections. The adjacent alternate serial sections were subjected to immunostaining with $\mathrm{CB}$ antibody for labeling of the entire mossy fiber projection. The procedure for labeling sections with $\mathrm{CB}$ antibody is the same as applied for sections used in the immunoelectron microscopy preparation. The level of Timm's staining versus CB labeling in adjacent sections was compared via a normalization method used in a previous study (Bagri et al., 2003).

DiI labeling of mossy fibers. Wild-type P15 mice were anesthetized with an overdose of Nembutal and perfused transcardially with cold saline, followed by $4 \%$ paraformaldehyde in $0.1 \mathrm{~m}$ phosphate buffer. Brains were postfixed several hours in $4 \%$ paraformaldehyde in $0.1 \mathrm{M}$ phosphate buffer at $4^{\circ} \mathrm{C}$, and $500 \mu \mathrm{m}$ slices of tissue containing dorsal or ventral cross-sections of the hippocampus were cut using a Leica VT1000 S vibrating microtome. Pressurized microinjections of a $0.5 \%$ solution of DiI (Molecular Probes, Eugene, OR) in dimethylformamide were performed using a Picospritzer II system (Wilkerson, Englewood, CO). DiI injections were made in various regions of the infrapyramidal blade of the dentate gyrus, and the dye was allowed to trace for 1 week at $37^{\circ} \mathrm{C}$ in $4 \%$ paraformaldehyde solution. Tissue slices were resectioned on a vibrating microtome at a thickness of $50-100 \mu \mathrm{m}$ and imaged on a Zeiss 

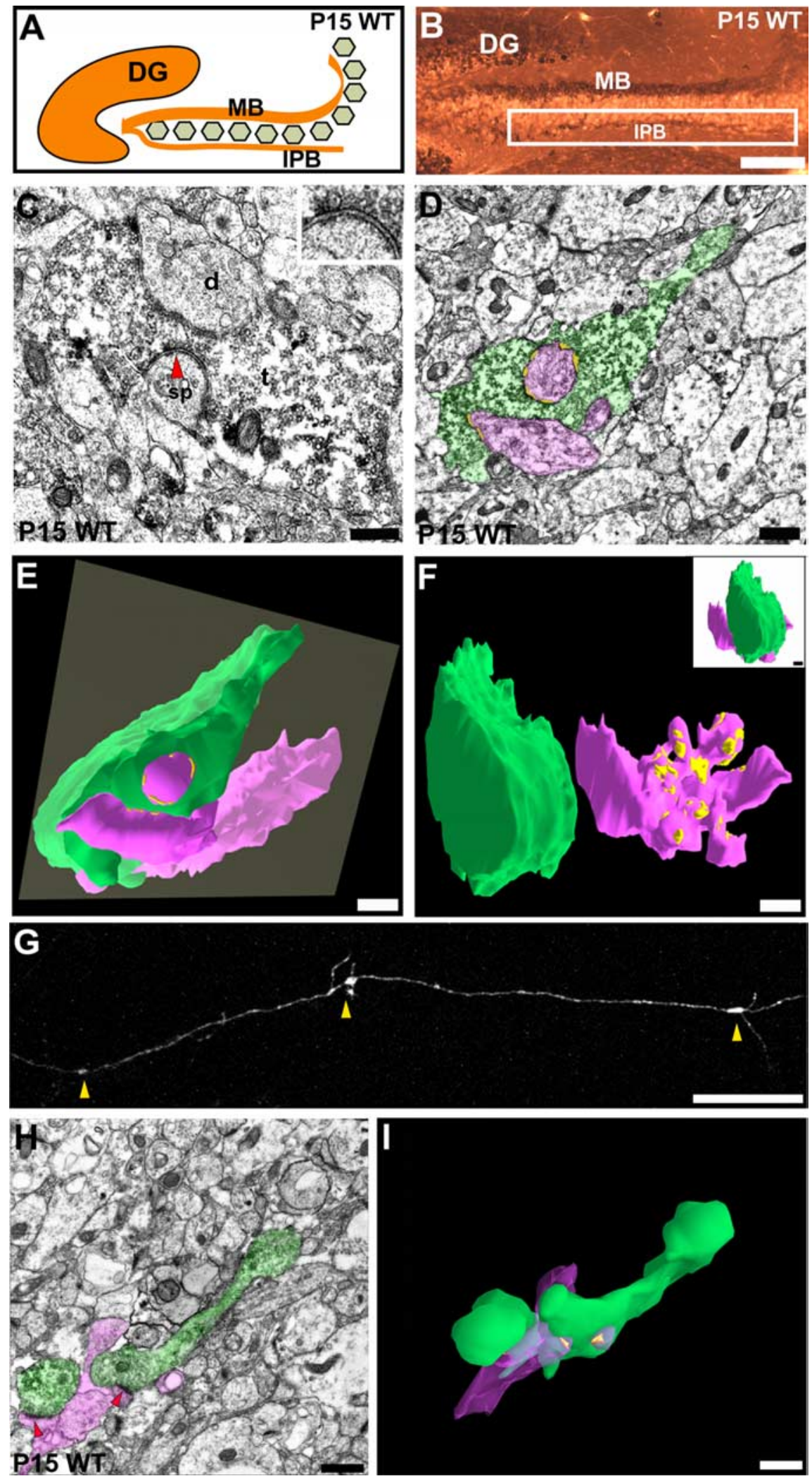

Figure 1. Synaptic complex formation in the IPB of WT mice before IPB pruning. $A, B$, Diagram and cross section of the dorsal hippocampus from a WT mouse at $P 15$ that was stained for an antibody for $C B$. The white square indicates a region of the IPB that was analyzed further with electron microscopy in C-F. DG, Dentate gyrus. C, Electron micrograph of a CB-immunolabeled bouton in the IPB of a WT mouse at P15. The mossy terminal-like bouton ( $\mathrm{t}$ ) forms asymmetrical synapses with a spine (sp) and a dendritic shaft (d). An asymmetric synapse on a spine (arrowhead) is shown in higher power in the inset. D, Electron micrograph of a larger P15 WT mossy fiber bouton (green) taken before pruning of the IPB. The bouton establishes several asymmetric synapses (yellow) with a dendritic shaft and its spines (pink). The micrograph was taken from one of 23 thin sections ( $70 \mathrm{~nm}$ per section) from the 3D serial EM reconstruction shown in $\boldsymbol{E}$ (gray square). $\boldsymbol{E}, 3 \mathrm{D}$ serial reconstruction of the same P15 WT mossy fiber bouton in association with
(Thornwood, NY) LSM 510 META laserscanning confocal microscope. Images were analyzed using Zeiss META software version $3.2 \mathrm{SPZ}$.

\section{Results}

Synaptic complexes form before hippocampal mossy fiber axons are pruned

The mossy fibers of the dentate gyrus innervate CA3 pyramidal cells in the hippocampus. In early postnatal mice, the dentate granule cells send an $\mathrm{MB}$ of projections that lie adjacent to the apical dendrites of CA3 pyramidal cells and a transient set of IPB axons that project to the CA3 basal dendrites (Bagri et al., 2003). $\mathrm{MB}$ axons form stable "synaptic complexes" in which a presynaptic mossy fiber bouton establishes multiple excitatory synapses with CA3 apical dendritic spines (as defined by Amaral and Dent, 1981). By later postnatal ages, our studies have shown that a spatially restricted expression of a local gradient of Sema3F acting through a receptor complex involving NPN-2 and PLXA3 drives the pruning of IPB axons back to the hilus of the dentate gyrus (Bagri et al., 2003). However, before the mossy fiber axons were removed from the IPB, it was unclear whether they formed synaptic complexes, because previous studies were all performed in the unpruned mature mossy fibers (Amaral and Dent, 1981; Seki and Rutishauser, 1998).

To address whether transient mossy fiber collateral projections from the IPB of the hippocampus establish synaptic connectivity with the basal dendrites of CA3 pyramidal cells, we used anti-CB immunoelectron microscopy to perform a detailed ultrastructural analysis of the subcellular changes occurring at the

a CA3 dendrite and multiple spines branching off of the dendritic shaft. $\boldsymbol{F}$, The mossy fiber bouton in $\boldsymbol{E}$ has been rotated $90^{\circ}$ and is separated from the postsynaptic elements to display the complex branching pattern of the dendritic spines that establish synaptic contacts (yellow patches) in the central portions of the bouton. G, A laser-scanning confocal microscopic image showing a distal segment of an IPB mossy fiber axon taken from a P15 brain slice that was labeled with Dil. The arrowheads point to en passant giant mossy fiber terminals along the labeled axon. $\boldsymbol{H}$, Electron micrograph of a CB-immunolabeled longitudinally cut mossy fiber axon from the IPB at P15. The axon (green) contains an en passant bouton that is filled with vesicles and forms asymmetrical synapses (arrowheads) with a dendritic spine and shaft (pink). $I$, Serial $3 \mathrm{D}$ reconstruction of the same axon segment (green) in $\boldsymbol{H}$. Multiple synapses (yellow patches) are present and associated with a dendritic shaft and spine (pink). Scale bars: $\boldsymbol{B}$, $150 \mu \mathrm{m} ; C, H, I, 0.2 \mu \mathrm{m}$, and for inset in $C$, same scale bar represents $0.1 \mu \mathrm{m} ; \boldsymbol{D}-\boldsymbol{F}, 0.5 \mu \mathrm{m} ; \mathbf{G}, 50 \mu \mathrm{m}$. 
presynaptic mossy fiber boutons before pruning at P15 (Fig. $1 A, B)$ in WT CD-1 mice (Bagri et al., 2003). CB is localized primarily within mossy fibers of the $\mathrm{MB}$ and IPB. Although a few interneurons are $\mathrm{CB}$ positive within the regions of interest at later developmental stages, most are located outside pathways coursed by $\mathrm{MB}$ and IPB mossy fibers; in addition, their terminals and synapses are morphologically distinguishable from typical mossy fiber synaptic complexes (Freund and Buzsaki, 1996).

At P15, dense labeling of mossy fiber boutons appeared throughout areas associated with the IPB. These boutons exhibited complex variations in shape and contained multiple mitochondria and numerous synaptic vesicles (Fig. 1C). Most importantly, pools of synaptic vesicles were present in the boutons adjacent to presynaptic membranes that formed typical synaptic contacts with the basal dendrites of the CA3 pyramidal cells. This demonstrates that synaptic complexes are clearly formed within the IPB before pruning of IPB mossy fiber axons occurs. These IPB synaptic complexes resemble MB mossy fiber synaptic connections with CA3 apical dendrites clarified in previous studies (Chicurel and Harris, 1992).

The complexity of a typical mossy fiber synaptic complex from the IPB at P15 was clearly evident from partial 3D reconstructions of two CB-immunolabeled mossy fiber boutons (Fig. $1 E, F$ ) (supplemental Fig. S1, available at www.jneurosci.org as supplemental material). One synaptic complex was reconstructed from 23 serial thin sections at a total thickness of 1.61 $\mu \mathrm{m}$ (Fig. 1D). The presynaptic bouton extended in multiple directions, whereas a large dendritic shaft with multiple-headed spines coursed through the center of the bouton. Multiple pools of synaptic vesicles in the arms of the mossy fiber bouton were present adjacent to numerous areas of synaptic contact with dendritic spines. From the detached $3 \mathrm{D}$ reconstruction image shown in Figure $1 F$, patch-like PSDs (yellow) were associated with several dendritic spine heads (pink) that branched from the dendritic shaft, indicating the presence of multiple synaptic release sites. Interestingly, typical IPB mossy fiber boutons at this age closely resembled mossy fiber boutons observed in the MB that were not pruned during development (Amaral and Dent, 1981; Chicurel and Harris, 1992).

\section{Mossy fiber synaptic complexes disappear during IPB pruning}

Although a few studies have reported evidence that different cellular mechanisms are involved in different forms of pruning [axosome shedding for short terminal arbors at the NMJ (Bishop et al., 2004) in vertebrates and axonal degeneration for stereotyped pruning (Watts et al., 2004; Marin et al., 2005) in Drosophila], none have reported whether the same mechanisms were involved for stereotyped pruning in the vertebrate CNS. To address this, we analyzed in detail the ultrastructural changes that occurred during pruning from P20 to P35 (Fig. $2 A-F$ ) in the IPB using $\mathrm{CB}$ immunoelectron microscopy.

After P20, the ultrastructural features of mossy fiber synaptic complexes in the IPB changed dramatically as the mossy fiber axon collaterals were being pruned. At P25 and P35, the number of labeled mossy fiber boutons in the IPB subfield was decreased significantly $(3.93 \pm 0.21,1.37 \pm 0.24$, and $0.43 \pm 0.12$ boutons/ $100 \mu \mathrm{m}^{2}$ at P15, P25, and P35, respectively; $p<0.05$, ANOVA, Newman-Keuls test; $n=10$ adjacent areas in the IPB on two serial sections for each age). This was not surprising considering that a majority of the mossy fibers have been pruned back to the hilus of the dentate gyrus by these ages.

Using quantitative immunoelectron microscopy, we found significant ultrastructural changes in the mossy fiber synaptic complexes in the IPB at P25 and P35. The typical labeled IPB mossy fiber bouton at P25 (Fig. 2C) was much smaller in size (70\% decrease in perimeter compared with P15) (see Fig. 4B) and less complex compared with mossy fiber boutons at P15 (Fig. $1 C)$. Each bouton at P25 retained fewer synaptic contacts with CA3 dendrites (55\% fewer synapses) (Fig. 4C), yet a majority of IPB mossy fiber boutons ( $80 \%$ with one or more synapses; $n=15$ boutons) maintained some form of synaptic contact, either with a dendritic spine or, in most cases, a dendritic shaft. Interestingly, in the larger boutons that were present at P25 $(n=12$ boutons $>7 \mu \mathrm{m}$ in perimeter from $n=2$ animals), we noticed a significant decrease in the number of synapses compared with P15 boutons ( $n=15$ boutons $>7 \mu \mathrm{m}$ in perimeter from $n=2$ animals) that were of similar size $(0.91 \pm 0.19$ asymmetric contacts per bouton at P25 vs $3.80 \pm 0.38$ asymmetric contacts per bouton at P15; $p<0.01$, Student's $t$ test), suggesting that many of the synaptic contacts could be lost before the regression in bouton size.

The changes seen at P35 (Fig. $2 F$ ) when pruning was almost completed appeared more advanced than we observed at P25. Most IPB boutons were much smaller (70\% decrease in perimeter compared with $\mathrm{P} 15 ; n=10$ boutons) and less complex in shape. Indeed, at P35, a majority of the labeled boutons did not establish any identifiable synaptic contact $(n=6$ of 10 boutons without synapses). Together, this result indicates that there was a regression in the development of the mossy fiber synaptic complexes that was associated with the pruning of mossy fibers from the IPB.

\section{No evidence of degeneration and glial involvement during IPB pruning}

We next examined whether axonal degeneration was involved during pruning. We analyzed in serial EM sections the integrity of the plasma membranes and the fine structures of mossy fiber axons and boutons to detect any signs of axon degeneration in the entire IPB subfield that may not have been found using silver staining techniques in our previous study (Bagri et al., 2003). In serial thin sections ( $n=10$ sets of thin sections per mouse with shortened fibers in the IPB; $n=3$ mice per age at P20 and P25) of longitudinally cut axons and cross-sectioned mossy fiber boutons during pruning from P20 to P25 when shortening of the IPB occurred, we found that most if not all of the labeled axons and boutons in the IPB appeared normal (Fig. 2I,J). Their plasma membranes and cytoskeletal components including the neurofilaments and microtubules seemed intact and comparable with the profiles before pruning at P15 (Fig. $1 H, I$ ), except that the caliber of the axons were thinner $(0.23 \pm 0.01 \mu \mathrm{m}$ at P15, $n=14$ axons; $0.14 \pm 0.02 \mu \mathrm{m}$ at $\mathrm{P} 25, n=13$ axons; $p<0.005$, Student's $t$ test), suggesting that the axons were retracting.

Because no signs of degeneration were observed along the mossy fiber axons and their synaptic complexes, we assessed whether the presence of glial cells could explain the dramatic loss in synapse number and bouton size during pruning after P20. We used serial EM to investigate in more detail whether glial cells in the area were invading and surrounding immunolabeled IPB mossy fiber synaptic complexes as they were being pruned away between early and peak stages of pruning from P20 to P25. Serial EM reconstructions (all serial reconstructions spanned up to 1.5 $\mu \mathrm{m}$ in thickness) of CB-immunolabeled small IPB boutons at P20 ( $n=4$ representative boutons of 20 that were fully reconstructed) and P25 ( $n=5$ representative boutons of 20 that were fully reconstructed) were prepared from CB-immunolabeled tissues in which the IPB was observed to be in the process of pruning 

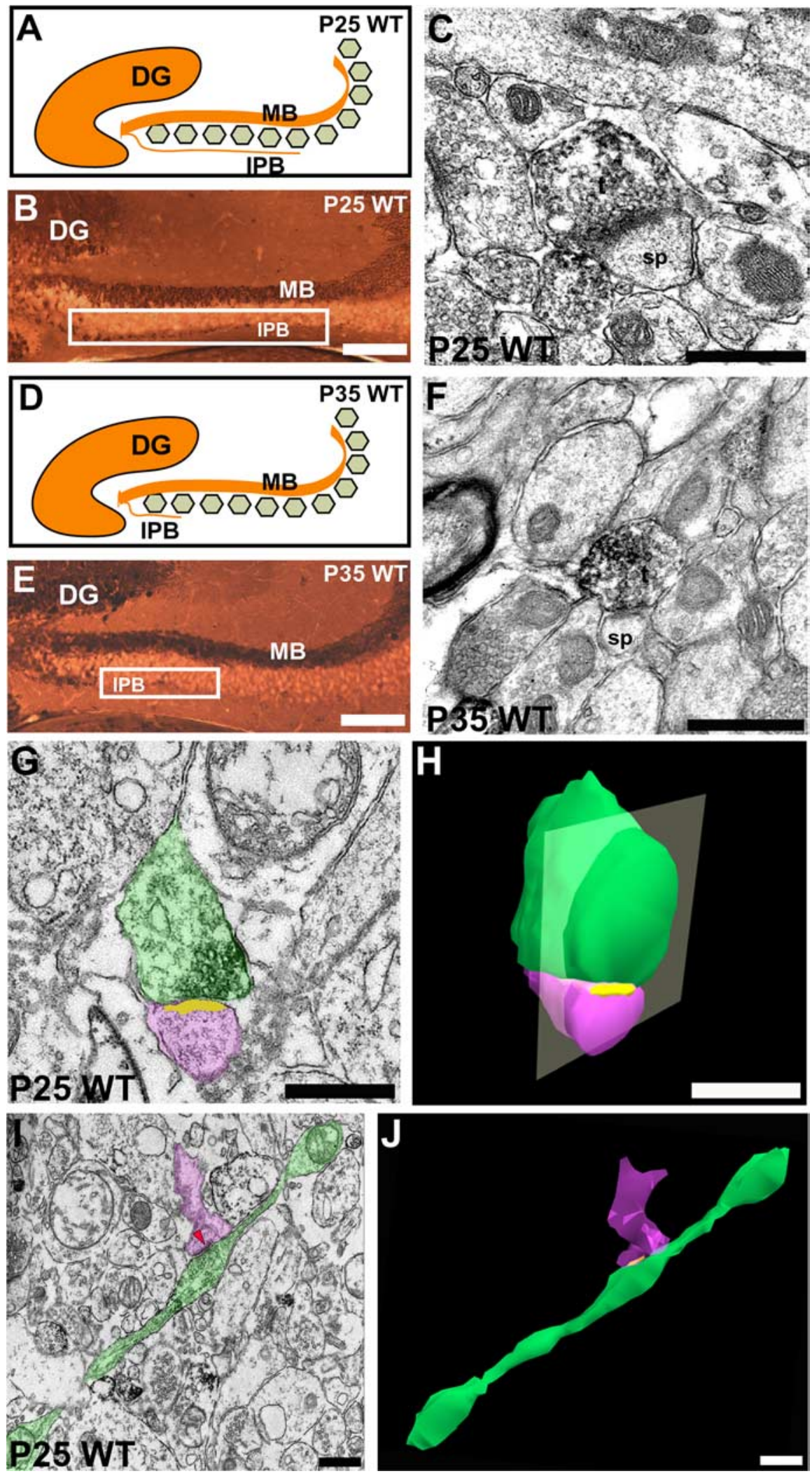

Figure 2. Synaptic complex elimination in the IPB of WT mice during IPB pruning. $A, B, D, E$, Diagrams and cross sections of the dorsal hippocampus from a WT mouse at $P 25$ and $P 35$ that were stained for an antibody for $C B$. The white squares indicate regions of the IPB that were analyzed further with electron microscopy for P25 $(\boldsymbol{C})$ and P35 (F). C, P25 IPB, a typical CB-immunolabeled bouton ( $\mathrm{t}$ ) is much smaller compared with boutons at $\mathrm{P} 15$ and retains only a single asymmetrical contact with a spine (sp). $\boldsymbol{F}, \mathrm{P} 35$ IPB, a smaller CB-immunolabeled bouton (t) retains only a single synapse with a spine (sp). $\mathbf{G}$, Electron micrograph of a small bouton (green) in contact (yellow) with a dendritic spine (pink) taken during pruning of the IPB from a P25 WT mouse (1 of 12 thin sections; $70 \mathrm{~nm}$ per section). $\boldsymbol{H}, 3 \mathrm{D}$ serial reconstruction of the same mossy fiber bouton in $\boldsymbol{G} . \mathbf{I}$, An electron micrograph (1 of 6 thin sections, $70 \mathrm{~nm}$ per section) showing a longitudinally cut, (B-immunolabeled axon (green) and one of its en passant boutons taken
(Fig. 2G,H) (supplemental Fig. S1, available at www.jneurosci.org as supplemental material). Interestingly, we found that the presynaptic boutons and postsynaptic elements were more or less intact, and, in most cases, only a single PSD was identified in synaptic complexes that were completely reconstructed. These results suggest that the functional coupling sites between mossy fiber boutons and the basal dendrites of CA3 pyramidal neurons were dramatically reduced during IPB shortening. After carefully examining the serial sections and the reconstructed synaptic complexes, no evidence of axon degeneration and glial cell engulfment was observed associating with the labeled synaptic complexes. Based on recent results for pruning of short axon collaterals at the NMJ (Bishop et al., 2004), one interpretation could be that the smaller boutons were remnants that were derived from a much larger presynaptic mossy fiber bouton. Two lines of evidence, however, would argue against this view. In most cases (19 of 20 boutons analyzed for 3D reconstruction), the smaller boutons were not in close proximity to a larger bouton at P20 (an earlier stage of pruning at which larger boutons were present), as was observed at the mammalian NMJ (Bishop et al., 2004). Second, although in rare cases (1 of 20 boutons analyzed for 3D reconstruction) glial cell processes were found to be either adjacent to or pass by the synaptic complexes, no glial cell processes were observed invading synaptic regions occupied by smaller boutons or distal axon segments that lacked a synaptic contact (data not shown). Together, these results support a mechanism in which synaptic complex and collateral process elimination occurs via a retraction process as opposed to Wallerian degeneration or distal to proximal axosome shedding that has been reported from previous studies (Jones and Powell, 1969; Bishop et al., 2004; Marin et al., 2005).

\section{$\leftarrow$}

from a P25 WT mouse during IPB pruning. Microtubules and neurofilaments are still abundant in the axon, but the bouton has regressed in size and is seen containing diffusely distributed vesicles and retaining only one asymmetric synaptic contact (arrowhead) on a dendritic spine that buds off of a small dendritic shaft (pink).J, Serial 3D reconstruction of the same axon in $I$. The axon (green) is shown with its bouton synapsing (yellow) onto a dendritic spine (pink). Note that the axon caliber is narrower compared with axons at P15 (see Fig. $1 H, I)$. The bouton is much smaller than at P15 and is shown here retaining only one asymmetric synaptic contact with a dendritic spine. Scale bars: $\boldsymbol{B}, \boldsymbol{E}, 150 \mu \mathrm{m} ; \boldsymbol{C}, \boldsymbol{F}, \boldsymbol{I}, \boldsymbol{J}, 0.2$ $\mu \mathrm{m} ; \boldsymbol{G}, \boldsymbol{H}, 0.5 \mu \mathrm{m}$. DG, Dentate gyrus. 


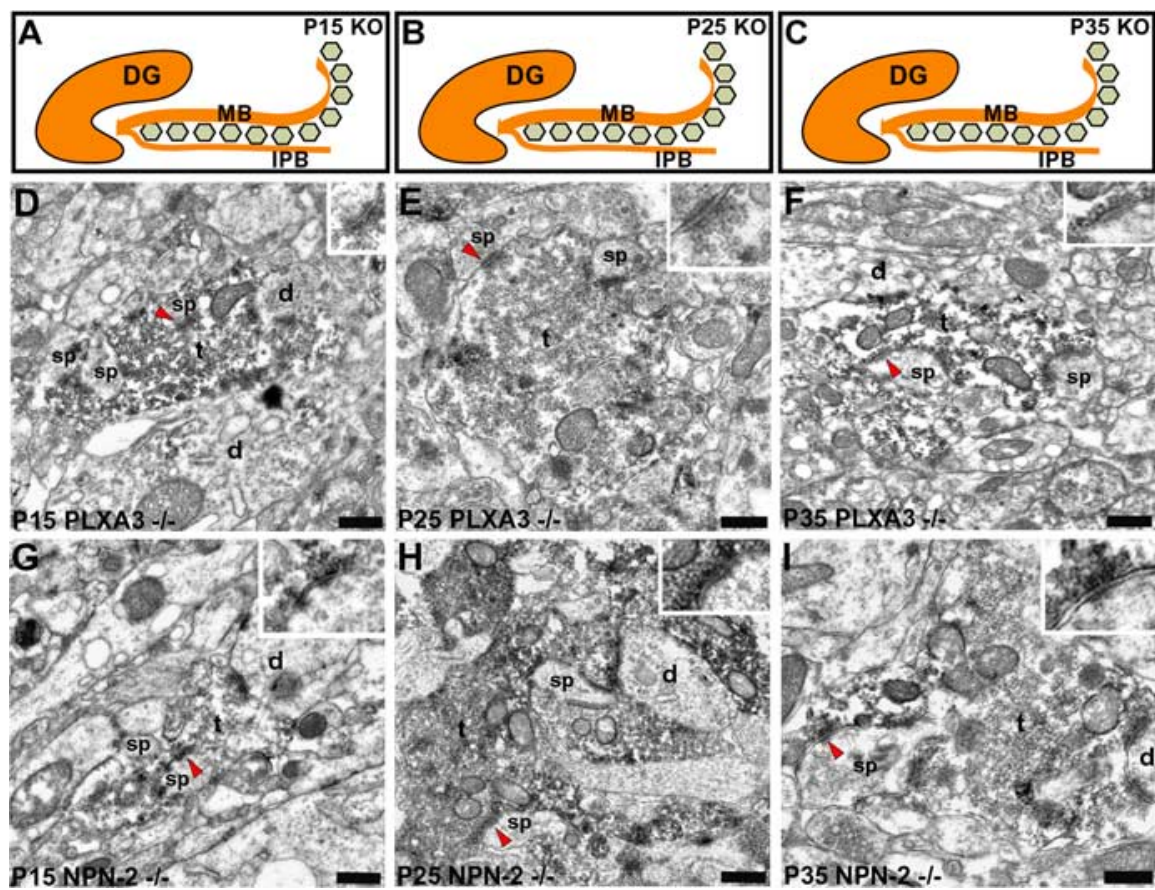

Figure 3. Loss of PLXA3 signaling results in continued maturation of synaptic complexes in the IPB of PLXA3 and NPN-2 knock-out mice. $A-C$, Diagram demonstrating the pruning defect observed for mossy fibers in the IPB of $P L X A 3^{-/-}$and NPN$2^{-/-}$mice at P15 (A), P25 (B), and P35 (C). D-F, Immunoelectron micrographs show the ultrastructural features of CBimmunolabeled mossy terminal-like boutons (t) in the IPB at different postnatal ages of $P L X 3^{-/-}$animals. The arrowheads point to asymmetric contacts on spines shown in higher power in the insets. Scale bars: $0.2 \mu \mathrm{m}$; insets, same scale bar represents 0.1 $\mu \mathrm{m} . \mathbf{G}-\mathrm{I}$, Immunoelectron micrographs show similar ultrastructural features of CB-immunolabeled, mossy terminal-like boutons (t) in the IPB at different postnatal ages of $N P N-2^{-/-}$animals; note that some boutons form multiple asymmetrical contacts with spines (sp) and dendrites (d). The arrowheads point to asymmetric contacts on spines shown in higher power in the insets. Scale bars: $0.2 \mu \mathrm{m}$; insets, same scale bar represents $0.1 \mu \mathrm{m}$. DG, Dentate gyrus.

\section{Loss of PLXA3 and NPN-2 signaling results in continued but normal synaptic complex development in the IPB}

In $P L X A 3^{-/-}$and NPN-2 $2^{-/-}$CD-1 mutant mice (Chen et al., 2000; Cheng et al., 2001; Bagri et al., 2003), a pruning defect persists because mossy fiber projections in the IPB cannot respond to a local Sema3F gradient, and, as a result, the projections do not prune back to the hilus of the dentate gyrus (Fig. $3 A-C$ ). We reasoned that, if PLXA3 signaling initiated pruning by first perturbing the further development of the mossy fiber synaptic complex, then the inability to respond to a local Sema3F gradient by knocking out either coreceptor, PLXA3 or NPN-2, should result in the continued maturation of the mossy fiber synaptic complexes in the IPB. To confirm this hypothesis, we studied the ultrastructure of developing mossy fiber synaptic complexes from the IPB of PLXA3 $3^{-/-}$and NPN-2 $2^{-/-}$mice from ages P15 to P35 using the same approach as in the WT mice.

Before pruning at P15, the IPB synaptic complexes of $P L X A 3^{-/-}$and NPN-2 $2^{-/-}$mice (Fig. 3D, G) were indistinguishable from the IPB synaptic complexes observed in P15 WT mice. At P25 and P35, the IPB mossy fiber synaptic complexes of $P L X A 3^{-/-}$and $N P N-2^{-/-}$mice continued to develop. The ultrastructural changes that arise during the development of the synaptic complexes in the MB (which is not pruned) have been well documented (Amaral and Dent, 1981). Interestingly, the development of mossy fiber synaptic complexes in the IPB of both mutant mice was consistent with the development of mossy fiber synaptic complexes observed within the $\mathrm{MB}$ of knock-out and wild-type mice (Fig. $3 E, F$ and $H, I$ ) (Amaral and Dent, 1981). In the absence of PLXA3 signaling, the IPB mossy fiber boutons continued to acquire more complex structures. They were larger and more complex in shape, and they established more synaptic contacts with dendritic spines on both the peripheral surface and within the central portions of the mossy fiber bouton (Fig. $4 A-$ $C)$. Our results indicate that mossy fiber synaptic complex elimination requires PLXA3 signaling. The absence of PLXA3 signaling allows mossy fiber synaptic complexes to continue to develop normally like mossy fiber synaptic complexes in the WT MB.

A quantitative study of mossy fiber synaptic complex development in the IPB highlighted some key differences in development between the WT and PLXA3 ${ }^{-1-}$ and $N P N-2^{-1-}$ mossy fiber profiles. We used a staging paradigm (see Materials and Methods) based on a previous study (Amaral and Dent, 1981) from WT MB synaptic complexes to classify different degrees of developmental maturity for each synaptic complex. The analysis revealed significant differences between distributions of mossy fiber synaptic complexes at later stages of development in WT versus $P L X A 3^{-1-}$ and $N P N-2^{-1-}$ mice (Fig. 4A). Age-matched comparisons at age P15 showed no significant difference in mossy fiber synaptic complex distribution between WT versus $P L X A 3^{-/-}$and NPN-2 $2^{-/-}$mice. However, by ages P25 and P35, the distribution was shifted toward less mature synaptic complexes in WT mice, whereas in both mutant mice the majority of synaptic complexes continued to develop toward more mature appearing profiles. A more detailed analysis of features such as the average size of mossy fiber bouton, average number of synaptic contacts, and average number of spines associated with each mossy fiber bouton demonstrated that additional differences existed within age-matched comparisons between P25 and P35 WT versus P25 and P35 PLXA3 $3^{-1-}$ and NPN-2 $2^{-1-}$ mice (Fig. $4 B-D$ ). In both knock-out phenotypes, we found that IPB mossy fiber synaptic complexes were clearly more developed, because they tended to be significantly larger in size and established more synaptic contacts with the spines of CA3 pyramidal neurons (Fig. $4 B-D)$. Interestingly, we found no significant differences between the average lengths of the PSDs for WT versus age-matched $P L X A 3^{-/-}$and $N P N-2^{-/-}$mossy fiber boutons (Fig. $4 E$ ).

\section{Synaptic complexes that are formed in the IPB before pruning} contain components of functional synapses

To further characterize the development of the synaptic complexes in the IPB, we analyzed the synaptic complexes for the presence of presynaptic and postsynaptic molecular markers (presynaptic, VGLUT1 and VGLUT2; postsynaptic, NMDAR1) (Malenka and Nicoll, 1993; Petralia et al., 1994; Nicoll and Malenka, 1995; Fremeau et al., 2001, 2004a,b). Unexpectedly, we found that VGLUT1 and VGLUT2 were both expressed in the developing mossy fibers (as observed in the MB at P15) (Fig. $5 A, B$ ) (supplemental Fig. S2, available at www.jneurosci.org as 
supplemental material), but only VGLUT1 was expressed in the adult fibers (as observed in the MB at P45) (Fig. 5C,D) (supplemental Fig. S2, available at www. jneurosci.org as supplemental material). The expression of VGLUT2 was downregulated when the synaptic complexes became morphologically mature (stage 3 ) (Fig. 5D). These results have been confirmed in recent published studies of VGLUT expression in the hippocampus (Fremeau et al., 2004a): the authors demonstrated that VGLUT1 and VGLUT2 were both required for distinct activities and that VGLUT1-positive adult mossy fibers transiently express VGLUT2 early in development. Thus, we were able to link the expression of two VGLUTs to a relative degree of maturation for mossy fiber development; that is, coexpression of VGLUT1 and VGLUT2 indicated immature mossy fibers early in development, whereas expression of only VGLUT1 indicated more mature mossy fibers in adults.

Before pruning in the IPB of WT, $N P N-2^{-/-}$, and $P L X A 3^{-/-}$P15 mice, the positive expression of VGLUT1 and VGLUT2 within mossy fiber boutons implied that these synaptic complexes were still developmentally immature (Fig. $5 A, B, G$ ) (supplemental Fig. S2, available at www.jneurosci.org as supplemental material). In contrast, in the most developed synaptic complexes from the IPB of knock-out mice at P45, only VGLUT1 expression was observed presynaptically (Fig. $5 E, F, I$ ). In addition, we observed the presence of positive NMDAR1 expression in postsynaptic sites associated with IPB mossy fiber synaptic complexes in WT and knock-out mice at different stages of development of the IPB from P15 to P45 (Fig. $5 H, J$ ) (supplemental Fig. S2, available at www.jneurosci.org as supplemental material). These findings indicate that IPB synaptic complexes can develop normally like $\mathrm{MB}$ synaptic complexes if they are not eliminated by PLXA3 signaling, as we observed in $\mathrm{NPN}-2^{-/-}$and $P L X A 3^{-/-}$ mice.

\section{Intact PLXA3 signaling allows for the elimination of synaptic complexes before mossy fiber axon collateral pruning}

If PLXA3 signaling was responsible for initiating the pruning of mossy fibers by preventing the further maturation of synaptic complexes, we reasoned that the gradual loss of synapses must occur before the pruning of mossy fibers for support of a retraction phenomenon absent of any signs of degeneration. Preliminary data
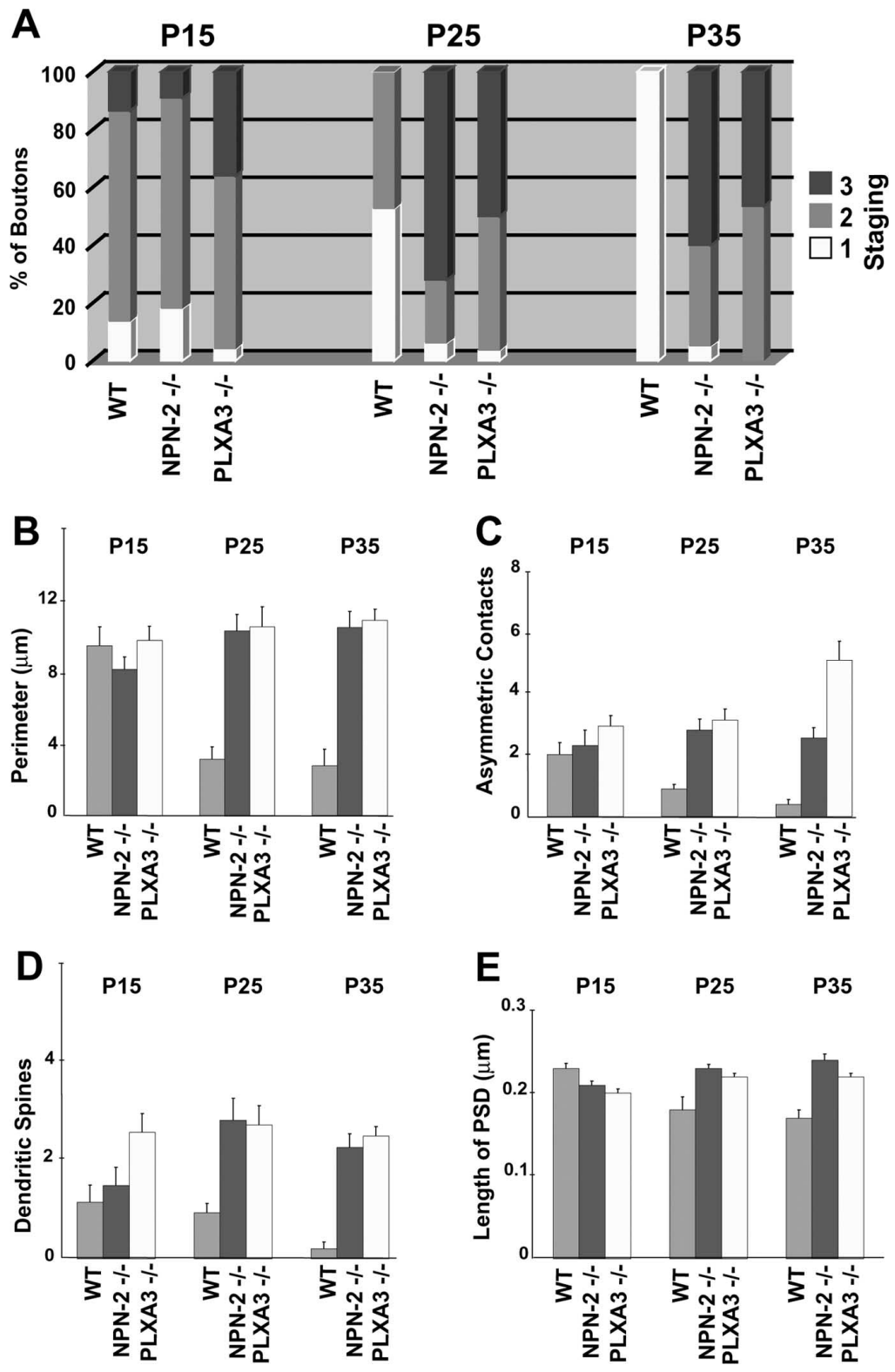

Figure 4. Quantification of mossy fiber synaptic complexes from WT, NPN-2 $2^{-/-}$, and $P L X A 3^{-/-}$mice. $A$, Bar graph showing the percentage distribution of mossy fiber synaptic complexes for each phenotype (WT, $N P N-2^{-/-}$, and $P L X 3^{-/-}$) in the IPB at three different postnatal ages. Stage 1 synaptic complexes (white) are classified as containing relatively small and immature mossy fiber boutons with one or no synaptic contacts. Stage 2 synaptic complexes (gray) are classified as intermediate in complexity and contain mossy fiber boutons that establish more synaptic contacts on dendritic shafts than with spines. Stage 3 synaptic complexes (black) contain the most mature and complex mossy fiber boutons that often establish a majority of their synapses on dendritic spines. Staging parameters were modified from a previous study (Amaral and Dent, 1981) (see Materials and Methods). Significant differences between means determined for each grouping were observed for P15 WT versus P25 WT, P15 WT versus P35 WT, and P25-P35 WT versus age-matched P25-P35 NPN-2 ${ }^{-1-}$, and PLXA3 ${ }^{-1-}$ samples ( $p<0.05$; ANOVA, Newman-Keuls test). $\boldsymbol{B}$, Bar graph showing the average perimeter (mean $\pm \mathrm{SE}$ ) of a mossy fiber bouton for each phenotype (WT, $N P N-2^{-\prime-}$, and $P\left(X A 3^{-\prime-}\right.$ ) at three different postnatal ages. Significant differences between means were observed only between P15 WT versus P25 WT, P15 WT versus P35 WT, and P25-P35 WT versus age-matched P25-P35 NPN- $2^{-1-}$ and PLXA3 ${ }^{-1-}$ samples $(p<0.05)$. C, Bar graph showing the average number (mean $\pm \mathrm{SE}$ ) of asymmetric contacts per bouton for each phenotype (WT, NPN-2 $2^{-1-}$, and $P L X 3^{-1-}$ ) at three different postnatal ages. Significant differences between means were observed only between P15 WT versus P35 WT and P25-P35 WT versus age-matched $N P N-2^{-/-}$and PLXA3 ${ }^{-/-}$samples ( $p<$ 0.05). D, Bar graph showing the average number (mean \pm SE) of dendritic spines associated with each mossy fiber bouton for each phenotype (WT, NPN-2 $2^{-/-}$, and $P L X 3^{-1-}$ ) at three different postnatal ages. Significant differences between means were observed only between P25-P35 WT versus age-matched P25-P35 NPN-2 $2^{-/-}$and PLXA3 ${ }^{-/-}$samples $(p<0.05)$. E, Bar graph showing the average PSD length (mean \pm SE) for mossy fiber boutons of each phenotype (WT, NPN- ${ }^{-/-}$, and $P L X 3^{-1-}$ ) at three different ages that formed synapses. No significant differences between means were observed in all comparisons of means for each sample $(p>0.10) . A-D, A$ minimum of 15 synaptic complexes were analyzed for each phenotype and age except for $P 35$ WT ( $n=10$ boutons) in which most synaptic complexes were already removed from the IPB. $\boldsymbol{E}$, A minimum of 30 PSDs were analyzed for each phenotype and age except for P25 WT ( $n=15$ PSDs) and P35 WT ( $n=4$ PSDs). 

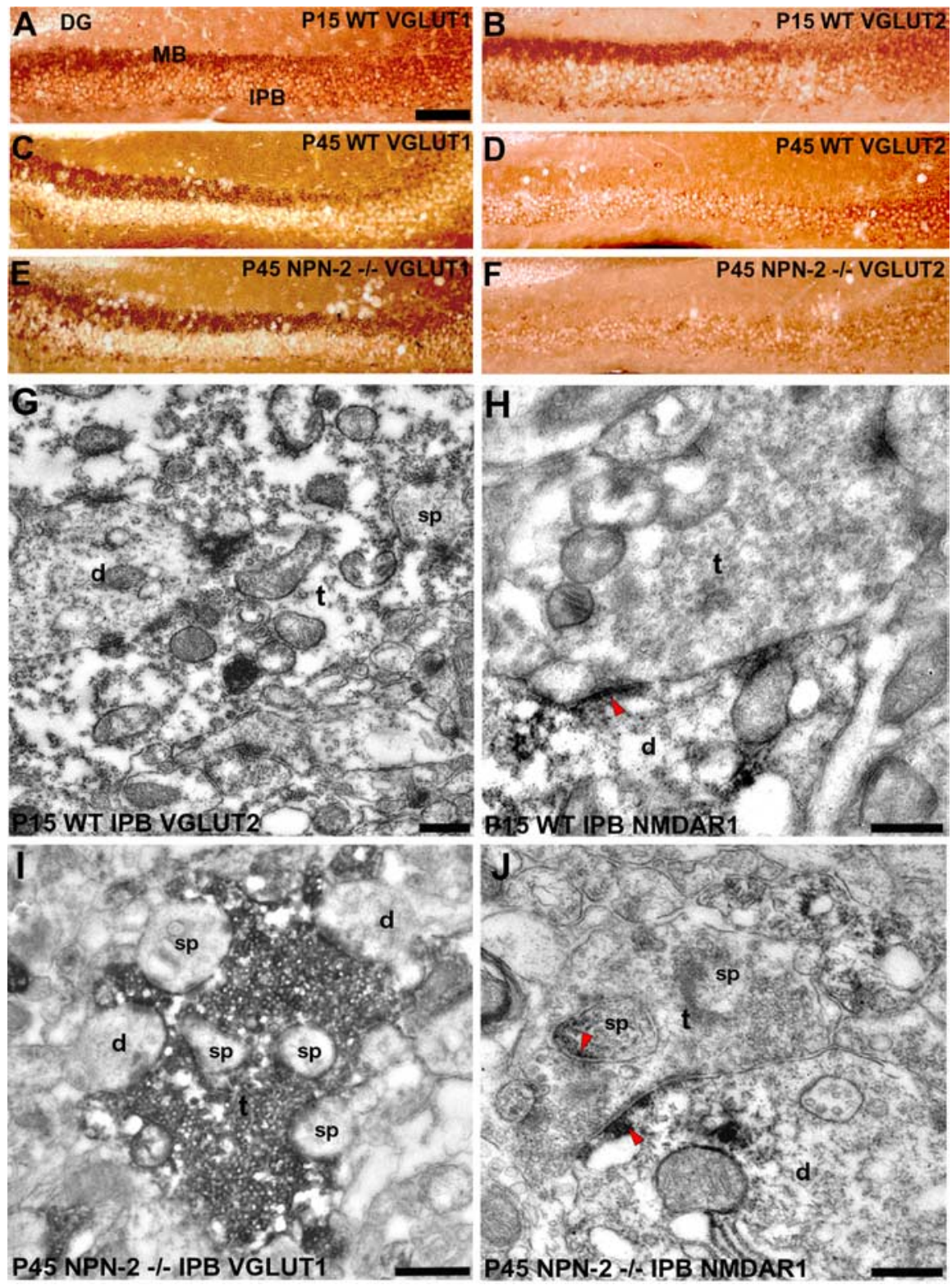

Figure 5. Characterization of mossy fiber synaptic complexes of the IPB with synaptic markers. Coronal sections of the dorsal hippocampus were immunostained with VGLUT1 $(\boldsymbol{A}, \boldsymbol{C}, \boldsymbol{E})$ and VGLUT2 $(\boldsymbol{B}, \boldsymbol{D}, \boldsymbol{F}) . \boldsymbol{A}, \boldsymbol{B}, \mathrm{P} 15$ WT hippocampi show positive labeling of the MB and IPB for both VGLUT1 and VGLUT2. C, D, P45 WT hippocampi show only positive labeling of the MB for VGLUT1. $E, F$, P45 NPN-2 $2^{-1-}$ hippocampi show only positive labeling of the IPB for VGLUT1. DG, Dentate gyrus. Scale bar: $\boldsymbol{A}-\boldsymbol{F}, 150 \mu \mathrm{m} . \mathbf{G}, \mathrm{P} 15$ WT, an immunoelectron micrograph shows VGLUT2 immunoreactivity in association with synaptic vesicles in a mossy terminallike bouton (t). The bouton forms multiple synaptic contacts with spines (sp) and a dendritic shaft (d) in the IPB. $\boldsymbol{H}$, P15 WT, an immunoelectron micrograph shows NMDAR1 immunoreactivity localized adjacent to postsynaptic densities (arrowhead) in a dendritic shaft (d), which are in contact with a mossy terminal-like bouton (t) in the IPB. I, P45 NPN-2-/- , a mossy terminal-like bouton (t) filled with dense VGLUT1-immunolabeled vesicles makes multiple synaptic contacts with spines (sp) and dendrites (d). $J$, P45 NPN-2 $2^{-/}$, a large bouton (t) makes several synaptic contacts with NMDAR1-immunolabeled dendrites (d) and spines (sp); note that PSDs are associated specifically with NMDAR1 immunoreactivity (arrowheads). Scale bars: $G-J, 0.2 \mu \mathrm{m}$.

electron microscopy, visualizing morphological changes in mossy fiber boutons and axons simultaneously during axon retraction was difficult. To address this issue, we performed an experiment with Timm's staining and $\mathrm{CB}$ immunohistochemistry in alternate serial and adjacent coronal sections containing dorsal regions of the hippocampus in WT mice from ages P15 to P30.

Previous studies have shown that Timm's staining for zinc ions can specifically label the presynaptic boutons of mossy fibers (Haug et al., 1971; Haug, 1975; Stirling and Bliss, 1978). In a similar manner, $\mathrm{CB}$ and zinc ions colocalize within mossy fiber boutons; an additional advantage with $\mathrm{CB}$ immunolabeling is that mossy fiber projections are clearly labeled as well. We tested the sensitivity of these procedures by using P15 WT and P25 PLXA3 knock-out mice as controls. In the unpruned IPB and MB in both P15 WT $(n=3)$ and P25 PLXA3 $(n=4)$ mice, Timm's staining and $\mathrm{CB}$ immunolabeling associated with the same regions within the IPB and MB in serial sections (Fig. $6 A, D$ ).

If the initial loss of synaptic boutons was present during an intermediate stage of pruning, we reasoned that the majority of Timm's staining for mossy fiber boutons should be eliminated first, whereas the $\mathrm{CB}$ immunolabeling for some mossy fiber projections should remain in the IPB. Therefore, we examined the Timm's and CB labeling of serial sections $(n=10$ sections per mouse) of the hippocampus in P20-P25 WT mice during pruning. Consistent with previous observations that pruning of the WT IPB occurred quickly and randomly between P20 and P30 and that only $23 \%$ of animals examined at these ages presented with an intermediate stage of pruning (Bagri et al., 2003), 5 of 20 P20-P25 WT mice showed the Timm's staining was lost before the complete loss of CB labeling from the IPB (Fig. 6B) (supplemental Fig. S3, available at www.jneurosci.org as supplemental material). As expected, in a condition in which pruning was nearer to completion (Fig. 6C) (supplemental Fig. S3, available at www.jneurosci.org as supplemental material) ( $n=3$ mice at P25), the level of

from our EM analyses was consistent with this assumption because mossy fiber synaptic complexes were less abundant in P25 and P35 WT mice even when mossy fibers were still present. Additionally, synaptic complexes that were present at P25 and P35 tended to be smaller in size and contained fewer synaptic contacts. Longitudinal sections of mossy fiber axons taken during pruning demonstrated a large regression in mossy fiber bouton size but less of a decrease in the caliber of the axon compared with mossy fiber boutons and axons before pruning, respectively. However, because of the low spatial resolution associated with
Timm's and CB immunolabeling in adjacent serial sections was approximately equal as some of these mossy fiber boutons established more mature synaptic complexes. Together with the EM data, the results suggest that PLXA3 signaling initiates the pruning of mossy fiber projections by first causing the elimination of mossy fiber synaptic complexes (Fig. 6E, Model).

\section{Discussion}

The stereotyped pruning of long axon collaterals in the vertebrate CNS is a widespread phenomenon that has been described for 
$>20$ years (Innocenti, 1981; Stanfield et al., 1982; O’Leary et al., 1990); until now, however, the cellular mechanisms were unknown. In particular, it was unclear whether long axon collaterals were capable of forming synaptic contacts and what cellular processes were involved in the loss of the synaptic contacts (if they formed) and their axons as they were eliminated. Using a CNS system in which the pruning of long axon collaterals can be clearly visualized, our results showed that transient long axon collaterals can establish synapses. The cellular process that resulted in the elimination of synapses and the regression of axon collaterals did not involve degeneration of the axon terminal or an essential engulfment of axonal processes by glial cells. In addition, the association of plexin-A3 signaling with the elimination of these synaptic complexes suggests a unique function to a family of receptors that has been connected primarily with axon repulsion.

\section{Transient long axon collaterals in the CNS are capable of establishing synaptic contacts}

Our EM results from the IPB before pruning clearly indicate that long axon collaterals can synapse onto CA3 dendritic shafts and spines to form a unique structure: a synaptic complex. Before pruning, these synaptic complexes were found to be immature in development, as evidenced by a previous staging classification for their morphology and the presence of both synaptic vesicle markers VGLUT1 and VGLUT2. The mossy fibers and synaptic complexes of the IPB were subsequently eliminated after pruning.

What is the fate of the long axon collaterals if they are not pruned away? It appears, at least from our developmental studies of $P L X A 3^{-/-}$and $N P N-2^{-/-}$mice that these axons can form synaptic complexes that are morphologically and functionally indistinguishable from synaptic complexes derived from early postnatal and adult wild-type mice. The normal development of these synaptic complexes is consistent with our observations on the normal development of the unpruned MB synaptic complexes based on morphological and molecular analyses with VGLUT1 and VGLUT2. Furthermore, in C57BL/6 mice, in which the IPB happens to be intrinsically long, our results (data not shown) have indicated that, in the absence of any factors that may influence the stereotyped pruning of the IPB, the mossy fibers are capable of establishing normal-appearing contacts as long as they reach their targets.

Our results are also consistent with previous studies that have focused on other contexts of pruning. At the NMJ, motor neurons that collateralize within the target region have been known
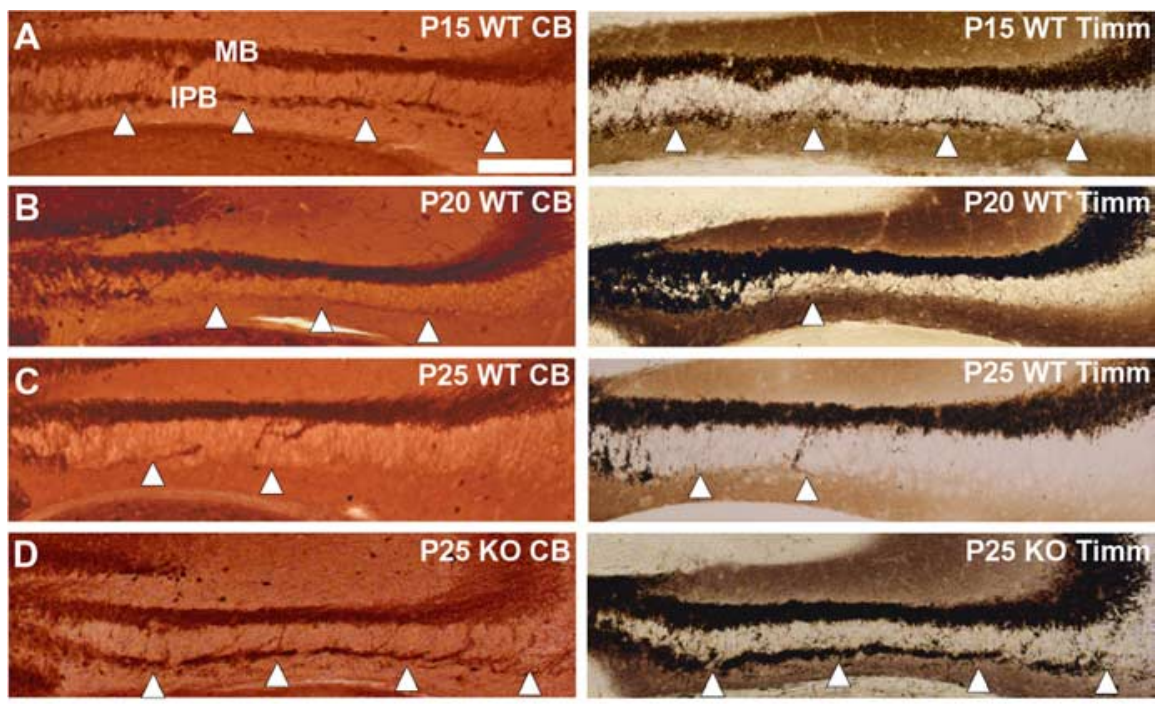

\section{E. Model for PLXA3 Dependent Stereotyped Axon Pruning}
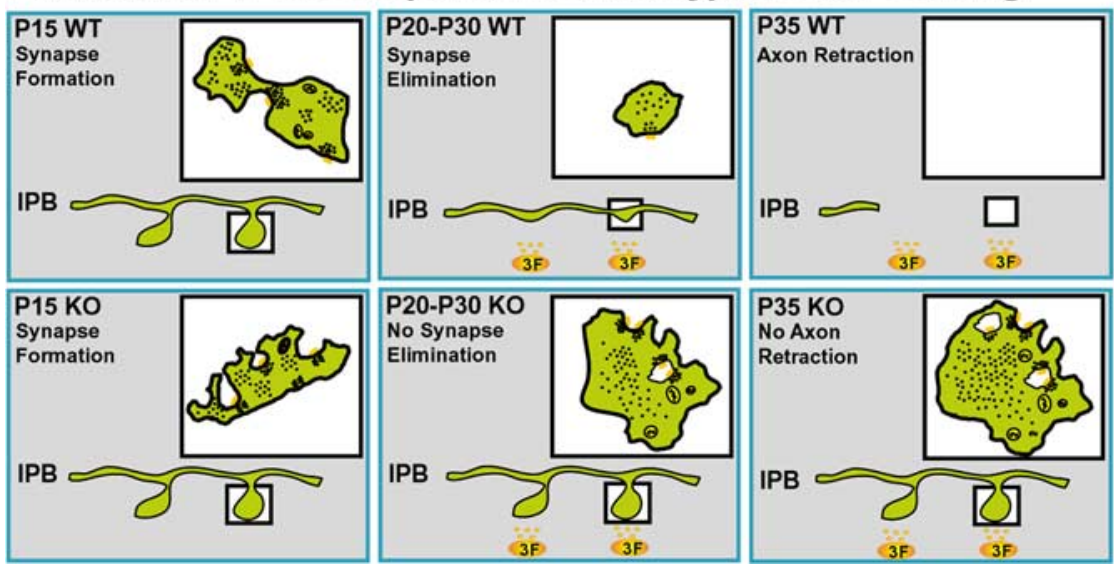

Figure 6. Synaptic complex elimination occurs before the pruning of mossy fibers in the IPB. A, P15 WT before the pruning of IPB mossy fibers. Coronal sections of dorsal hippocampi were immunostained for CB (left) for the entire mossy fiber projection and Timm's stained (right) for the mossy fiber boutons in the adjacent section. The level of staining of mossy fibers and boutons of the PB is approximately equal for CB and Timm's staining, respectively. B, P20WT, an intermediate stage of pruning in which some CB immunolabeling of mossy fibers persists (left), whereas the level of Timm's staining for mossy fiber boutons disappears (right). $\boldsymbol{C}$, P25 WT, a more completed stage of pruning shows that the level of CB and Timm's staining in the IPB is approximately equal, because the mossy fibers of the IPB have pruned back most of their transient projections to join the MB projections. D, P25 PLXA3 knock-out, a case in which pruning of the IPB does not occur and the levels of $C B$ and Timm's staining are approximately equal. The white arrowheads indicate the level of Timm's or CB labeling in the IPB. Scale bar: $\boldsymbol{A}-\boldsymbol{D}, 200 \mu \mathrm{m}$. $\boldsymbol{E}$, A schematic diagram illustrates synapses associated with en passant mossy fiber boutons that are organized periodically along the axon. In WT and KO (PLXA3 $3^{-1-}$ and $N P N-2^{-1-}$ ) mice, before pruning occurs, mossy fibers of the IPB (green) form immature synaptic complexes (insets, in left patches). During the process of pruning, intact PLXA3 signaling for Sema3F causes the gradual elimination of the synaptic complex top row, middle panel). The elimination of synaptic complexes leads to stereotyped pruning of the IPB (top row, right panel), whereas loss of PLXA3 signaling results in continued maturation of the synaptic complex (bottom row, middle and right panels) and a defect in IPB stereotyped pruning.

to establish functional contacts with multiple muscle fibers (Brown et al., 1976; Rosenthal and Taraskevich, 1977; Colman et al., 1997). A similar case has been described in the CNS for climbing fibers that provide collateral inputs to multiple Purkinje cells of the cerebellum before their inputs are pruned away (Crepel et al., 1976; Hashimoto and Kano, 2003).

Stereotyped pruning in the hippocampus occurs by retraction Knowledge of the idea that nervous systems of vertebrates and invertebrates establish an exuberant number of synapses via collateralization early in development has led many to speculate how 
the aberrant connections and their projections are removed by adulthood. Traditionally, axon retraction (Bixby and Spitzer, 1981; Riley, 1981) and Wallerian degeneration (Rosenthal and Taraskevich, 1977) were two popular models that had been proposed to account for the removal of exuberant axon collaterals in the CNS. In central neurons of Drosophila, a mechanism resembling classic Wallerian degeneration appears to mitigate the widespread stereotyped elimination of long axon collaterals (Zhai et al., 2003; Watts et al., 2004; Marin et al., 2005). And more recently, a third possible mechanism for axon removal has been proposed, axosome shedding, based on evidence from retracting short terminal arbors at the NMJ during synapse elimination (Bishop et al., 2004). However, no detailed study has documented whether the latter two mechanisms, Wallerian degeneration and axosome shedding, could account for the stereotyped loss of much longer axon collaterals in the vertebrate CNS.

In our analysis of the hippocampal mossy fiber system during pruning of the IPB, the results suggest a retraction phenomenon absent of degenerative-like causes that have been observed for the pruning of axons via axosome shedding and Wallerian degeneration. During axon retraction, it has been proposed that the microtubules and neurofilaments need to be intact for sufficient retrograde recycling of components from the axon terminal to other parts of the axon (Riley, 1981). Several lines of evidence from our EM analysis during pruning of the IPB can support this view. First, larger mossy fiber boutons that are still present during pruning seem to have less organelles and synapses, supporting the notion that the components of the mossy fiber bouton are being retrogradely transported elsewhere. Second, the mossy fiber boutons are regressing in size and number during pruning without any signs of degeneration. The loss in mossy fiber boutons before the axons are removed was clearly seen in our Timm's and CB labeling study in which the boutons seem to disappear before axon pruning as evidenced by the initial loss of Timm's staining of mossy fiber boutons before CB staining of axons. Interestingly, consistent with the idea that the boutons were indeed regressing in size before the removal of the axon was the finding that longitudinally cut axons that contained calbindin-positive mossy fiber boutons were still in contact with CA3 dendrites that resembled small mossy fiber boutons usually seen at much earlier stages of development (Amaral and Dent, 1981). Third, in serial EM section analyses of calbindin-positive and longitudinally cut axons during pruning, the microtubule and neurofilaments of retreating axons are quite intact, ensuring that mechanisms for fast and slow retrograde transport were possible.

Glial cells seem to play a pertinent role in the other models of pruning. In both the axosome shedding and classic Wallerian degeneration of retreating axons, glial cells apparently envelope and engulf remnants of the axons and terminals that are in the process of pruning. In the hippocampus, however, we saw no evidence of an increase in glial cell participation in the stereotyped removal of long IPB axon collaterals (data not shown). In fact, during IPB pruning, we did not observe any obvious signs of degeneration or glial cell process invasion of the synaptic area or along retreating axons as they were being removed from the IPB. In other regions of the CNS, it has been suggested that glial cells may factor more importantly in the elimination of exuberant synapses. During peak periods of climbing fiber synapse elimination, for example, no obvious signs of degeneration are observed, but there is an increase in double membrane-bound terminals that seem to be surrounded and taken up by Bergmann glial cell processes (Eckenhoff and Pysh, 1979); presumably, this could be the equivalent of axosome shedding in process in the developing CNS.

\section{PLXA3 signaling is required for synaptic complex elimination and axonal pruning}

Although many studies have described the functions of semaphorins on plexins in axon guidance (Kolodkin, 1996; Fujisawa, 2004), our results are also the first to suggest a role for PLXA3 signaling in eliminating synaptic complexes in the vertebrate CNS. Loss-of-function mutations for the Sema3F coreceptors $P L X A 3^{-/-}$and $N P N-2^{-/-}$led to exuberant synaptic complex formation in the IPB. Additional evidence that PLXA3 signaling was critical for the removal of synaptic complexes was observed from loss-of-function studies for the PLXA3 ligand Sema3F (Sahay et al., 2003, 2005). Sema3 $F^{-/-}$mice display a similar pruning defect in the IPB. Also consistent with the idea that plexin signaling from semaphorins was important for synapse elimination was the finding that invertebrate semaphorins serve as inhibitory signals for synapse formation in Drosophila (Matthes et al., 1995; Godenschwege et al., 2002), in which the loss of these signaling pathways results in promiscuous synapse formation.

What is the influence of plexin signaling on the cellular aspects of stereotyped axon collateral pruning? Plexin signaling might directly influence stereotyped axon pruning events by causing local reorganization of the cytoskeleton in transient long axon collaterals; this, in turn, would cause the destabilization and elimination of structures associated with the synaptic complex. However, our results suggest another intriguing possibility, that plexin signaling leads to direct synaptic complex elimination and axon collateral pruning follows as a result of the inability to reestablish stable synaptic complexes, whereas plexin signaling is still present in the IPB (Fig. 6 E, Model). Among larger mossy fiber boutons at P25 (during pruning), we observed a considerable decrease in asymmetric contacts compared with similarly sized boutons at P15 (before pruning). This would suggest that the individual components of the synaptic complex were being removed before the removal of the axon. Additional support for initial synaptic complex removal was observed in serial $3 \mathrm{D}$ reconstructions of longitudinally cut axons in which we noticed that the axon caliber and cytoskeletal structures (neurofilaments and microtubules) were only slightly decreased, yet a majority of mossy fiber boutons along the axon had regressed on average almost threefold in size. This finding was also supported by the loss of Timm's staining of mossy fiber boutons throughout the IPB before axons that were immunolabeled with CB. In addition, Sema3F is present during and after pruning of the IPB is mostly completed, an indication that the signal might act not only to initiate synaptic complex elimination but it also might prevent any synaptic complexes from reestablishing contacts that would stabilize the axon from being pruned from the IPB. However, to further support these results, future studies will need to address whether plexin receptors are colocalized at synaptic sites before stereotyped axon pruning.

\section{References}

Amaral DG, Dent JA (1981) Development of the mossy fibers of the dentate gyrus. I. A light and electron microscopic study of the mossy fibers and their expansions. J Comp Neurol 195:51-86.

Bagri A, Cheng HJ, Yaron A, Pleasure SJ, Tessier-Lavigne M (2003) Stereotyped pruning of long hippocampal axon branches triggered by retraction inducers of the semaphorin family. Cell 113:285-299.

Bernstein M, Lichtman JW (1999) Axonal atrophy: the retraction reaction. Curr Opin Neurobiol 9:364-370.

Bishop DL, Misgeld T, Walsh MK, Gan WB, Lichtman JW (2004) Axon 
branch removal at developing synapses by axosome shedding. Neuron 44:651-661.

Bixby JL, Spitzer NC (1981) Ultrastructural observations on synapse elimination in neonatal rabbit skeletal muscle. J Neurocytol 10:81-100.

Broadie K (2004) Axon pruning: an active role for glial cells. Curr Biol 14:302-304.

Brown MC, Jansen JKS, Van Essen D (1976) Polyneuronal innervation of skeletal muscle in new-born rats and its elimination during maturation. J Physiol (Lond) 261:387-422.

Chalupa LM, Killackey HP (1989) Process elimination underlies ontogenetic change in the distribution of callosal projection neurons in the postcentral gyrus of the fetal rhesus monkey. Proc Natl Acad Sci USA 86:1076-1079.

Chen H, Bagri A, Zupicich JA, Zou Y, Stoeckli E, Pleasure SJ, Lowenstein DH, Skarnes WC, Chedotal A, Tessier-Lavigne M (2000) Neuropilin-2 regulates the development of selective cranial and sensory nerves and hippocampal mossy fiber projections. Neuron 25:43-56.

Cheng HJ, Bagri A, Yaron A, Stein E, Pleasure SJ, Tessier-Lavigne M (2001) Plexin-A3 mediates semaphorin signaling and regulates the development of hippocampal axonal projections. Neuron 32:249-263.

Chicurel ME, Harris KM (1992) Three-dimensional analysis of the structure and composition of $\mathrm{CA} 3$ branched dendritic spines and their synaptic relationships with mossy fiber boutons in the rat hippocampus. J Comp Neurol 325:169-182.

Colman H, Nabekura J, Lichtman JW (1997) Alterations in synaptic strength preceding axon withdrawal. Science 275:356-361.

Crepel F, Mariani J, Dehaye-Bouchard N (1976) Evidence for a multiple innervation of Purkinje cells by climbing fibers in the immature rat cerebellum. J Neurobiol 7:567-578.

Eckenhoff MF, Pysh JJ (1979) Double-walled coated vesicle formation: evidence for massive and transient conjugate internalization of plasma membranes during cerebellar development. J Neurocytol 8:623-638.

Fremeau Jr RT, Troyer MD, Pahner I, Nygaard GO, Tran CH, Reimer RJ, Bellocchio EE, Fortin D, Storm-Mathisen J, Edwards RH (2001) The expression of vesicular glutamate transporters defines two classes of excitatory synapse. Neuron 31:247-260.

Fremeau Jr RT, Kam K, Qureshi T, Johnson J, Copenhagen DR, StormMathisen J, Chaudhry FA, Nicoll RA, Edwards RH (2004a) Vesicular glutamate transporters 1 and 2 target to functionally distinct synaptic release sites. Science 304:1815-1819.

Fremeau Jr RT, Voglmaier S, Seal RP, Edwards RH (2004b) VGLUTs define subsets of excitatory neurons and suggest novel roles for glutamate. Trends Neurosci 27:98-103.

Freund TF, Buzsaki G (1996) Interneurons of the hippocampus. Hippocampus 6:347-470.

Fujisawa H (2004) Discovery of semaphorin receptors, neuropilin and plexin, and their functions in neural development. J Neurobiol 59:24-33.

Godenschwege TA, Hu H, Shan-Crofts X, Goodman CS, Murphey RK (2002) Bi-directional signaling by Semaphorin la during central synapse formation in Drosophila. Nat Neurosci 5:1294-1301.

Hashimoto K, Kano M (2003) Functional differentiation of multiple climbing fiber inputs during synapse elimination in the developing cerebellum. Neuron 38:785-796.

Haug FM (1975) On the normal histochemistry of trace metals in the brain. J Hirnforsch 16:151-162.

Haug FM, Blackstad TW, Simonsen AH, Zimmer J (1971) Timm's sulfide silver reaction for zinc during experimental anterograde degeneration of hippocampal mossy fibers. J Comp Neurol 142:23-31.

Innocenti GM (1981) Growth and reshaping of axons in the establishment of visual callosal connections. Science 212:824-827.

Jones EG, Powell TP (1969) An electron microscopic study of the mode of termination of cortico-thalamic fibres within the sensory relay nuclei of the thalamus. Proc R Soc Lond B Biol Sci 172:173-185.

Kantor DB, Kolodkin AL (2003) Curbing the excesses of youth: molecular insights into axonal pruning. Neuron 38:849-852.

Kolodkin AL (1996) Semaphorins: mediators of repulsive growth cone guidance. Trends Cell Biol 6:15-22.

Lee T, Marticke S, Sung C, Robinow S, Luo L (2000) Cell-autonomous re- quirement of the USP/EcR-B ecdysone receptor for mushroom body neuronal remodeling in Drosophila. Neuron 28:807-818.

Lichtman JW, Colman H (2000) Synapse elimination and indelible memory. Neuron 25:269-278.

Liu XB, Jones EG (2003) Fine structural localization of connexin-36 immunoreactivity in mouse cerebral cortex and thalamus. J Comp Neurol 466:457-467.

Lohof AM, Delhaye-Bouchaud N, Mariani J (1996) Synapse elimination in the central nervous system: functional significance and cellular mechanisms. Rev Neurosci 7:85-101.

Luo L, O'Leary DDM (2005) Axon retraction and degeneration in development and disease. Annu Rev Neurosci 28:127-156.

Malenka RC, Nicoll RA (1993) NMDA-receptor-dependent synaptic plasticity: multiple forms and mechanisms. Trends Neurosci 16:521-527.

Marin EC, Watts RJ, Tanaka NK, Ito K, Luo L (2005) Developmentally programmed remodeling of the Drosophila olfactory circuit. Development 132:725-737.

Matthes DJ, Sink H, Kolodkin AL, Goodman CS (1995) Semaphorin II can function as a selective inhibitor of specific synaptic arborizations. Cell 81:631-639.

Nicoll RA, Malenka RC (1995) Contrasting properties of two forms of longterm potentiation in the hippocampus. Nature 377:115-118.

O'Leary DD (1987) Remodelling of early axonal projections through the selective elimination of neurons and long axon collaterals. Ciba Found Symp 126:113-142.

O’Leary DD, Bicknese AR, De Carlos JA, Heffner CD, Koester SE, Kutka LJ, Terashima T (1990) Target selection by cortical axons: alternative mechanisms to establish axonal connections in the developing brain. Cold Spring Harb Symp Quant Biol 55:453-468.

Petralia RS, Wang YX, Wenthold RJ (1994) The NMDA receptor subunits NR2A and NR2B show histological and ultrastructural localization patterns similar to those of NR1. J Neurosci 14:6102-6120.

Purves D, Lichtman JW (1980) Elimination of synapses in the developing nervous system. Science 210:153-157.

Riley DA (1981) Ultrastructural evidence for axon retraction during the spontaneous elimination of polyneuronal innervation of the rat soleus muscle. J Neurocytol 10:425-440.

Rosenthal JL, Taraskevich PS (1977) Reduction of multiaxonal innervation at the neuromuscular junction of the rat during development. J Physiol (Lond) 270:299-310.

Sahay A, Molliver ME, Ginty DD, Kolodkin AL (2003) Semaphorin 3F is critical for development of limbic system circuitry and is required in neurons for selective CNS axon guidance events. J Neurosci 23:6671-6680

Sahay A, Kim CH, Sepkuty JP, Cho E, Huganir RL, Ginty DD, Kolodkin AL (2005) Secreted semaphorins modulate synaptic transmission in the adult hippocampus. J Neurosci 25:3613-3620.

Sanes JR, Lichtman JW (1999) Development of the vertebrate neuromuscular junction. Annu Rev Neurosci 22:389-442.

Seki T, Rutishauser U (1998) Removal of polysialic acid-neural cell adhesion molecule induces aberrant mossy fiber innervation and ectopic synaptogenesis in the hippocampus. J Neurosci 18:3757-3766.

Stanfield BB, O'Leary DD, Fricks C (1982) Selective collateral elimination in early postnatal development restricts cortical distribution of rat pyramidal tract neurones. Nature 298:371-373.

Stirling RV, Bliss TV (1978) Hippocampal mossy fiber development at the ultrastructural level. Prog Brain Res 48:191-198.

Watts RJ, Hoopfer ED, Luo L (2003) Axon pruning during Drosophila metamorphosis: evidence for local degeneration and requirement of the ubiquitin-proteasome system. Neuron 38:871-885.

Watts RJ, Schuldiner O, Perrino J, Larsen C, Luo L (2004) Glia engulf degenerating axons during developmental axon pruning. Curr Biol 14:678-684.

Weimann JM, Zhang YA, Levin ME, Devine WP, Brulet P, McConnell SK (1999) Cortical neurons require Otx1 for the refinement of exuberant axonal projections to subcortical targets. Neuron 24:819-831.

Zhai Q, Wang J, Kim A, Liu Q, Watts R, Hoopfer E, Mitchison T, Luo L, He Z (2003) Involvement of the ubiquitin-proteasome system in the early stages of Wallerian degeneration. Neuron 39:217-225. 